\title{
Observaciones y perspectivas sobre las modalidades de educación básica en América Latina en la era pandémica y pospandémica -Estudio de caso: Aprende en Casa de México
}

\section{Observations and Perspectives on Basic Education Modalities in Latin America in the Pandemic and Post-Pandemic Era. -Case Study: Aprende en Casa of Mexico}

\author{
Chen Xing \\ Universidad de Estudios Extranjeros de Guangdong. Guangdong, China \\ chenxing@gdufs.edu.cn \\ Zhao Xi \\ Universidad de Estudios Extranjeros de Guangdong. Guangdong, China \\ 20180501954@gdufs.edu.cn
}

\section{Resumen:}

La crisis sanitaria de covid-19 ha llevado a que el modelo educativo en muchos países, incluidos los latinoamericanos, pase a la modalidad remota. Este artículo tiene como objetivo explorar la transición de la presencialidad a la virtualidad a lo largo de la pandemia, así como sus implicaciones para las posibilidades y disponibilidades pedagógicas en la era pospandémica mediante un análisis de la modalidad de la educación básica en América Latina basándose en un estudio de caso del programa educativo a distancia en México: Aprende en Casa.

Las conclusiones sugieren que el sistema mexicano de educación básica ha experimentado tres etapas de transformación ante la pandemia. Sin embargo, también observamos que en el proyecto Aprende en Casa existen varias deficiencias, vinculadas principalmente a la falta de garantía de equidad y eficiencia educativa. Se finaliza concluyendo que, con la finalidad de abordar los retos en la educación básica tanto en México como en toda la región latinoamericana, hace falta una visión hacia un enfoque integral, atendiendo la diversidad de las condiciones socioeconómicas.

Palabras claves: covid-19, educación básica, educación a distancia, transformación, desafíos

RED. Revista de Educación a Distancia. Núm. 67, Vol. 21. Artíc. 9, 30-Sep-2021

DOI: http://dx.doi.org/10.6018/red.480841 
RED. Revista de Educación a Distancia. Núm. 67, Vol. 21. Artíc. 9, 30-Sep-2021

DOI: http://dx.doi.org/10.6018/red.480841

\begin{abstract}
:
The Covid-19 health crisis has led to a shift in the educational model in many countries to the remote mode, including Latin American countries. This article aims to analyse the modality of basic education in Latin America based on a case study of the distance education programme in Mexico: Learning at Home. In this article, the objective is to explore the transition from face-to-face education to virtual mode, as well as its implications for pedagogical and public proposals in the post-pandemic era.

The findings suggest that the Mexican basic education system has undergone three stages of transformation in the face of the pandemic. However, we also note that there are several shortcomings in the Learning at Home project, mainly linked to the lack of guarantee of educational equity and efficiency. We conclude that in order to address the challenges in basic education not only in Mexico, but also in the Latin American region as a whole, a revolutionary vision towards a comprehensive approach is needed, taking into account the diversity of socio-economic conditions.
\end{abstract}

Key words: Coivd-19, basic education, distance education, transformation, challenges

\title{
1. Introducción
}

Nuevos brotes del coronavirus de 2020 siguen teniendo lugar en todo el mundo, y todos los sectores de la vida cotidiana se enfrentan a importantes retos. La educación no es una excepción. Según un informe de la UNESCO (Durand, 2020), 1.300 millones de niños y adolescentes de edad escolar de todo el mundo se han visto afectados por el nuevo coronavirus y, hasta noviembre de 2020, las suspensiones educativas a nivel mundial siguen afectando a 500 millones de alumnos, entre los cuales un 30\% está en América Latina. Siendo una de las regiones más afectadas por la pandemia en el mundo, la continuidad y el desarrollo de la educación básica tanto en una crisis sanitaria pública como en la poscrisis resultan un gran desafío para los países latinoamericanos. En este contexto pandémico han salido a la luz diversos modelos educacionales de emergencia, y "Aprende en Casa" de México es uno de los más representativos en el territorio latinoamericano. Lo tomamos como caso de estudio a partir de dos consideraciones: por un lado, aunque México se cuenta entre los países con mayor número de muertes por la pandemia en el mundo, en esta crisis ha establecido un mecanismo de respuesta nacional relativamente más sistemático y sistémico que tiene mayor relevancia para el modelo regional de educación

Observaciones y perspectivas sobre las modalidades de educación básica en América Latina en la era pandémica y pospandémica-Estudio de caso: Aprende en Casa de México. Xing y Xi. 
básica; por otro lado, a pesar de ser un país geográficamente grande y más desarrollado dentro de la región, se caracteriza por extremas desigualdades en el desarrollo social, también en el campo educativo, las cuales han sido y son más relevantes durante la pandemia. Por razones arriba mencionadas, el modelo educativo aplicado en México merece una especial atención para el estudio de la dinámica de la educación básica en América Latina en el contexto de la nueva pandemia.

\section{Marco contextual: educación básica de México y sus}

\section{problemas}

Según la Clasificación Internacional Normalizada de la Educación (CINE) (UNESCO, 1976), establecida por la UNESCO en 1976, la educación básica se refiere a la educación formal primaria y al primer ciclo de secundaria y es la educación más básica necesaria para la supervivencia humana en la sociedad moderna en comparación con la educación superior. Sin embargo, cómo se entiende este concepto varía considerablemente de un país a otro. En el caso de México, como resultado de la reforma del artículo III de la Constitución Política de los Estados Unidos Mexicanos en 2002, la educación preescolar pasó a formar parte oficialmente del sistema de educación básica. Y a partir de ese momento, el sistema mexicano de educación básica, que incluye la preescolar, la primaria y la secundaria, ha ido tomando forma.

En el siglo XX, el Gobierno mexicano ha ido reconociendo el papel clave de la educación en el desarrollo del país y ha ido mejorando el sistema educativo nacional mediante una serie de políticas. A mediados y finales del siglo XX, una serie de reformas, entre ellas el Undécimo Plan Anual, contribuyeron a aumentar la proporción del gasto público en educación, a mejorar la calidad de la enseñanza y a sistematizar la educación para todos los ciudadanos. En 2013, el Plan Nacional de Desarrollo 2013-2018, emitido por el Gobierno de Nieto, incluyó la educación como uno de los cinco principales objetivos de desarrollo del país y reiteró como uno de los derechos humanos fundamentales el derecho a la educación, del que gozan todos los ciudadanos de los Estados Unidos Mexicanos; el informe también prestó especial atención a la mejora de la calidad de la enseñanza y destacó el papel de la tecnología moderna en la educación (SEP, 2013).

La educación básica actual en México se caracteriza por los siguientes rasgos:

- Es estatal, con la educación pública como eje y la privada como complemento.

- El sistema educativo es jerárquico y sistemático.

- Está incluida en el sistema de educación obligatoria y, como bien público y gratuito, evoluciona como un desarrollo universal y socialmente inclusivo.

Observaciones y perspectivas sobre las modalidades de educación básica en América Latina en la era pandémica y pospandémica-Estudio de caso: Aprende en Casa de México. Xing y Xi. 
- Su desarrollo es valorado por el Estado y va ganando terreno en la estrategia nacional de desarrollo y en el sistema de protección del bienestar social.

Sin embargo, el sistema de educación básica de México también adolece de una serie de problemas. En primer lugar, la calidad general de la educación, en la que se centra la estrategia de desarrollo del Gobierno, sigue sin ser prometedora a pesar de las numerosas reformas. Los datos del Programa para la Evaluación Internacional de Alumnos (PISA), llevado a cabo por la Organización para la Cooperación y el Desarrollo Económico (OCDE) cada tres años, desde 2000 hasta 2018, entre adolescentes de 15 años de 60 a 80 países del mundo, muestran que los resultados de los alumnos mexicanos en tres materias (lectura, matemáticas y ciencias) están muy por debajo del promedio de los estudiantes de otros países participantes y, en su mayoría, en el rango medio-inferior.

En segundo lugar, la cobertura nacional de la educación básica en México antes de la pandemia aún estaba lejos de alcanzar el objetivo de la universalización para todos los ciudadanos. En 2019, la cobertura de la educación básica del país fue del 98,7\% para la educación primaria, el $84 \%$ para la secundaria y solo el $71,8 \%$ para la preescolar (SEP, 2020). Y, según datos del Instituto Nacional de Geografía y Estadística de México (INEGI) correspondientes a 2015, la tasa nacional de analfabetismo de la población mayor de 15 años es del 5,5\%, lo que equivale a 4 millones de personas en el país (INEGI, 2015).

Las desigualdades arriba mencionadas en materia de educación también se reflejan en la distribución de infraestructuras escolares. Según el informe de la Secretaría de Educación Pública de México, publicado en 2019, el 29\% y el 13\% de las escuelas tienen problemas de suministro de agua y de luz respectivamente, y casi la mitad carece de equipamiento informático y el 40\%, de internet. Esta escasez de infraestructuras agrava, sin duda, el desfase en la calidad educativa en la dimensión nacional.

La repentina alteración por la pandemia ha tenido un impacto significativo en la educación. Al mismo tiempo que los problemas inherentes al sistema de educación básica mexicano y los antes mencionados han quedado expuestos e incluso se han magnificado durante este período particular del coronavirus, nuevos problemas han surgido con la implantación de modelos educativos provisionales.

\section{Modalidad de educación básica en México en la pandemia}

A medida que la pandemia se ha ido profundizando, la modalidad educativa de México está pasando por un proceso de mejora continua, dividida en las siguientes tres etapas principales:

Observaciones y perspectivas sobre las modalidades de educación básica en América Latina en la era pandémica y pospandémica-Estudio de caso: Aprende en Casa de México. Xing y Xi. 


\subsection{Modelo informal de emergencia}

El 28 de febrero de 2020 se notificó en México el primer caso del coronavirus, y las autoridades declararon la primera fase de prevención y control del brote. Sin embargo, el cierre oficial de los centros educativos y la suspensión urgente de la enseñanza presencial no se anunciaron hasta el 20 de marzo, fecha del comienzo de la segunda fase de prevención. Tres días después, mientras el país entró en un período de tres meses de cuarentena domiciliaria, el sistema de educación básica empezó a adoptar un modelo de educación a distancia, modelo como respuesta del sistema educativo a la emergencia sanitaria a nivel nacional e internacional. Dado su carácter contingente, informal y espontáneo, no existía un marco formal de orientación establecido por el Ministerio de Educación, sino un programa desarrollado en colaboración con los maestros representantes.

\subsection{Aprende en Casa I}

El 21 de abril del mismo año las autoridades mexicanas anunciaron que el país entraba en la tercera fase de control de la pandemia; al mismo tiempo, la Secretaría de Educación de México lanzó el programa nacional de educación a distancia Aprende en Casa I. En vista de la alta tasa de televisores en el hogar (92,5\%) en comparación de la baja tasa del 44,3\% de implantación de ordenadores y del 56,4\% de cobertura de Internet en casa, Educación decidió utilizar la televisión como el principal vehículo de la enseñanza dirigida al alumnado de preescolar, primaria y secundaria, junto con la plataforma de Internet, la radio y los manuales de aprendizaje como herramientas auxiliares. Para las zonas marginadas que carecen de cobertura televisiva y radiofónica, el Consejo Nacional de Fomento Educativo (CONAFE) se encargó de distribuir regularmente manuales de enseñanza a domicilio y realizar visitas domiciliarias dos veces por semana para realizar un seguimiento del aprendizaje de los alumnos.

La brecha digital y los inconvenientes de la modalidad remota empiezan a manifestarse, contribuyendo a una diferenciación educativa importante en el contexto pandémico, cuya demostración directa es la variación de canales educativos a través de los cuales se llevan a cabo las actividades pedagógicas. Según la encuesta que realizó la Comisión Nacional para la Mejora Continua de la Educación (Mejoredu) de México con la finalidad de conocer las experiencias y aprendizajes vividos en la contingencia sanitaria, en esta etapa, un $65.5 \%$ de los estudiantes encuestados utilizan plataformas en línea como Google for Education; un 45,3\% aprovechan los recursos de Aprende en Casa I por internet, mientras que solo el $40.8 \%$ siguen la transmisión televisiva.

El factor tecnológico también ha tenido su impacto en el desempeño estudiantil. De acuerdo con una encuesta realizada en el estado de Nuevo León (México), los docentes perciben que casi la mitad de sus estudiantes han tenido dificultades en el manejo Observaciones y perspectivas sobre las modalidades de educación básica en América Latina en la era pandémica y pospandémica-Estudio de caso: Aprende en Casa de México. Xing y Xi. 
tecnológico. A esta línea se suma la falta de equipos y internet que les complica el aprendizaje remoto (García-Leal, etc., 2021).

En esta etapa, el modelo mexicano de educación básica bajo la pandemia tiene cierta formalidad por haber sido organizado por la Secretaría de Educación Pública e impartido en un formato fijo en el marco general pedagógico; sin embargo, no se puede ignorar su carácter también contingente: está diseñado para completar el semestre restante, siendo el contenido principal un repaso y consolidación de los conocimientos impartidos de forma presencial en el ciclo escolar 2019-2020. En general, es esencialmente un modelo pedagógico de emergencia en el corto plazo en respuesta a las circunstancias inesperadas, que cubre un corto período de tiempo y carece de objetivos a largo plazo.

Desde un punto de vista pragmático, el 59.6\% de los estudiantes de la primaria encuestados consideran que el programa ha cumplido su objeto de consolidar aprendizajes previos y, entre los de la secundaria, el $44.1 \%$. En cuanto al aprendizaje de nuevos conocimientos sobre sus materias, un 53\% de los de la primaria han dado una respuesta positiva, frente al 42.7\% de los de la secundaria (Comisión Nacional para la Mejora Continua de la Educación, 2020). Se puede aseverar, por ende, que Aprende en Casa I recibe una valoración modesta respecto al rendimiento pedagógico teniendo en cuenta su finalidad principal y los retos que tienen un vínculo directo a la brecha digital y la falta de habilidad tecnológica en el ámbito pedagógico.

\subsection{Aprende en Casa II}

El 3 de agosto de 2020, la Secretaría de Educación Pública de México anunció que para el ciclo escolar 2020-2021 se aplicaría el programa Aprende en Casa II que se caracteriza por su modelo híbrido continuando el modelo de educación televisada de Aprende en Casa I, con un programa en línea estandarizado a nivel nacional y complementado con instrucciones semipresenciales en regiones donde la pandemia está efectivamente contenida.

Cabe destacar que, en esta fase, además del plan de estudios tradicional, el Ministerio de Educación ha creado dos asignaturas para el programa televisivo "Vida saludable" y otra específica para los padres. Esta última está diseñada para guiar a los tutores de casa en la adaptación a este modelo educativo no convencional, mientras que la primera es un producto del marco educativo adaptado a las nuevas circunstancias pandémicas, cuyo objetivo es animar a los alumnos a hacer ejercicio, mejorar su sistema inmunológico y comer de forma saludable para prevenir y combatir la pandemia.

En tanto, la diferenciación en las herramientas pedagógicas como consecuencia de la dificultad para tener acceso a internet también se viene acentuando. Según una encuesta

Observaciones y perspectivas sobre las modalidades de educación básica en América Latina en la era pandémica y pospandémica-Estudio de caso: Aprende en Casa de México. Xing y Xi. 
realizada en el estado de Sonora (México) entre el mes de mayo a octubre, a los que no tienen acceso a internet les quedan el programa de televisión, los materiales de papel y las llamadas telefónicas para sostener la continuidad escolar mientras que los que lo tienen gozan de la posibilidad de utilizar las plataformas de comunicación simultánea y las redes sociales como medios principales, dejando así en un lugar de segundo plano Aprende en Casa que se transmite en televisión (Campa Álvarez, 2021).

En comparación con las primeras dos fases, Aprende en Casa II es altamente formal e institucionalizado, aunque es la continuidad consecuente de Aprende en Casa I, por lo que está oficialmente reconocida como equivalente a la educación tradicional presencial, proporcionando la posibilidad de que el modelo de educación híbrido se convierta en la nueva norma en la era pandémica y pospandémica. Por otra parte, después de los primeros dos intentos, el último ha adaptado el sistema de retroalimentación de la enseñanza y ha optimizado la eficacia y la eficiencia de la educación.

Sin embargo, en esta etapa, los problemas adaptativos relacionados con la transición a una modalidad remota se hacen cada vez más evidentes con el correr de los meses. Entre ellos figura la ausencia de un sistema formal de diagnóstico, en el sentido en que los maestros siguen los criterios tradicionales sin tener en cuenta las habilidades que surgen durante esta contingencia, por ejemplo, la colaboración familiar y el uso de las herramientas digitales. Tampoco existe un mecanismo comunicativo universal para atender las dudas de los alumnos de manera sincrónica. Por otra parte, hay reclamos desde la comunidad docente de que urgen instrucciones y capacitación por parte de la SEP a fin de adaptar el modelo de diseño a la altura de la planeación académica en un contexto virtual (Correa Terán. J, 2021).

\section{Deficiencias y retos del modelo de educación básica mexicano en el contexto de la pandemia}

A través de la descripción anterior, podemos ver que el gobierno mexicano, en respuesta a la interrupción de la educación convencional provocada por el covid-19, ha establecido gradualmente un modelo educativo híbrido, basado en la educación a distancia y complementado por la educación presencial en regiones y según el nivel de contención de la pandemia en cada zona. Sin embargo, la misma investigación llevada a cabo por GarcíaLeal et al. demostró: i) entre los maestros encuestados el 34,7\% planteó no haber visto los programas "Aprende en casa por TV"; ii) los docentes encuestados identificaron siete retos en cuanto al uso de la Tecnología Educativa: planeación del proceso de enseñanza $(5,8 \%)$, uso de medios y recursos tecnológicos $(5,8 \%)$, evaluación del proceso de aprendizaje $(18,8 \%)$, tolerancia y comprensión de las necesidades de los estudiantes $(3,5 \%)$, uso de la

Observaciones y perspectivas sobre las modalidades de educación básica en América Latina en la era pandémica y pospandémica-Estudio de caso: Aprende en Casa de México. Xing y Xi. 
computadora $(4,8 \%)$, comunicación con los estudiantes $(22,1 \%)$ y comunicación con los padres de familia o tutores $(12,0 \%)$; iii) dentro de los problemas pedagógicos, se identificó notable dificultad en la evaluación de las actividades $(55,7 \%)$, el tener un espacio inadecuado para trabajar desde casa $(41,6 \%)$ y dificultad en la planeación de actividades $(18,1 \%)$; etc. El cambio brusco y radical social ha caracterizado a esta adaptación educativa por la incertidumbre y la inseguridad, y en el transcurso del análisis también nos hemos dado cuenta de que dicha modalidad adolece de ciertos defectos institucionalizados y se enfrenta a retos de escepticismo social y dificultades en la aplicación.

\subsection{Diferenciación entre las garantías de equidad educativa}

El marco conceptual propuesto por Imke Harbers y Abbey Steele (2020) define la heterogeneidad territorial como las diferencias regionales en la provisión de bienes públicos y la protección de derechos por parte del Estado, la cual se clasifica en una tipología cuádruple de acuerdo con el tiempo y el espacio.

Uniformidad territorial

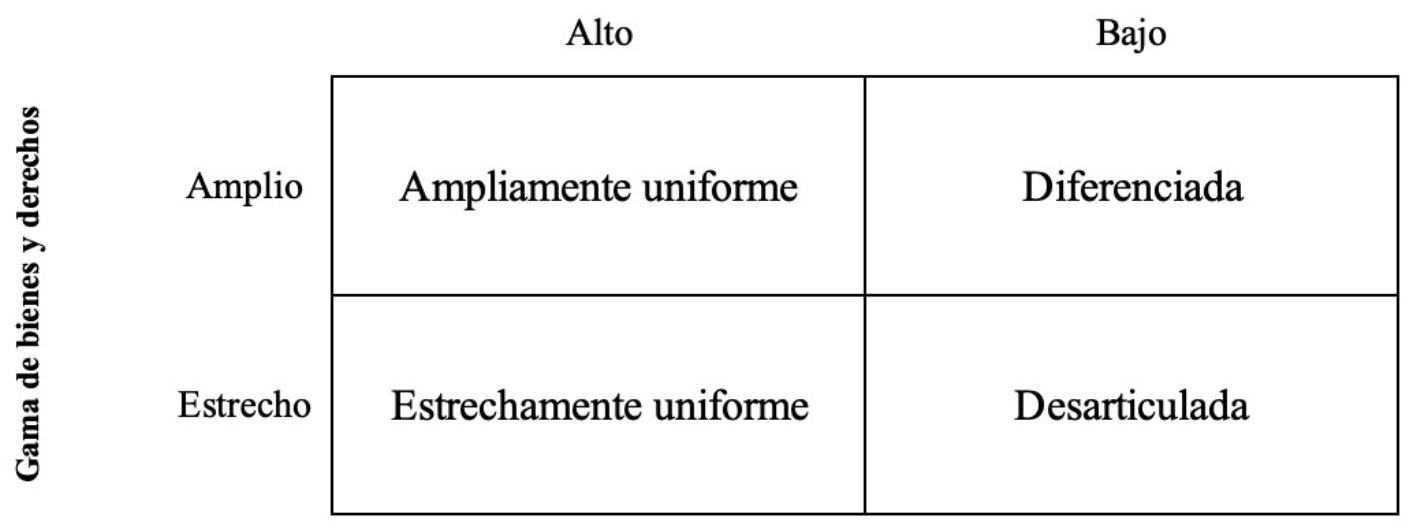

Fig.1 Tipología conceptual de los estados (Harbers \& Steele, 2020)

La educación básica pública en México, que es obligatoria y se imparte durante la pandemia a través de radio, televisión, etc., es un servicio prestado por el Gobierno y tiene la naturaleza de bien público. Por lo tanto, el marco anterior es aplicable a este caso.

El análisis basado en el marco se divide en dos perspectivas: la protección por parte del Estado del derecho a la educación de los alumnos en edad escolar y los bienes relacionados con la educación que proporciona el propio Estado.

Observaciones y perspectivas sobre las modalidades de educación básica en América Latina en la era pandémica y pospandémica-Estudio de caso: Aprende en Casa de México. Xing y Xi. 
En la primera perspectiva, un buen indicador es la tasa de abandono escolar en las circunstancias pandémicas en las que las condiciones económicas de las familias han sido duramente golpeadas por la recesión general. Según los datos más recientes (INEGI, 2020), en el ciclo 2020-2021, son 3 millones los estudiantes pertenecientes a la educación básica que dejaron la escuela, mientras que en agosto de 2020, SEP declaró que, tan solo entre los años escolares 2019-2020 y 2020-2021, las matriculaciones de la escuela primaria habían disminuido aproximadamente en 114.900 y de secundaria, en 9.500, lo cual representa un $10 \%$ de tasa de deserción bajo el impacto del covid-19 (Ríos, 2020). La modalidad educativa en la era pandémica no ha tocado los factores socioeconómicos subyacentes que garantizan los derechos de los alumnos.

Según la encuesta que realizó la Mejoredu (2020), las razones de exclusión de la educación a distancia ordenadas de acuerdo con el porcentaje de docentes que las mencionó son: i) falta de acceso a internet $(84,6 \%)$; ii) carencia de dispositivos electrónicos para acceder a las actividades (76,3\%); iii) escasez de recuersos económicos $(73,3 \%)$, lo cual revela que la penuria financiera y la brecha digital son las causas más relevantes al expulsar a los alumnos de la vida escoalr.

Las dos fases del programa Aprende en Casa muestran cierta heterogeneidad regional en la oferta de productos educativos, entre los cuales lo primero y más importante son los métodos y el seguimiento de los maestros. Aunque el Gobierno mexicano ha optado por programas de televisión de alta cobertura como principal medio de enseñanza, en realidad existen diferencias regionales en la elección de los equipos de aprendizaje. Como se puede observar en la Figura 2, las condiciones básicas (Internet, computadores, teléfonos móviles) para la enseñanza en las plataformas en línea muestran grandes diferencias geográficas de cobertura entre los estados mexicanos: Baja California tiene un $60,6 \%$ y un $83,9 \%$ de disponibilidad de computadoras y teléfonos móviles en los hogares respectivamente, en comparación con el $24,1 \%$ y $58,1 \%$ de Chiapas; en cuanto a Internet, la diferencia es de más del $80 \%$ frente al 24,6\%. En la figura siguiente, podemos ver que esta diferencia se da principalmente entre los estados del norte y del sur: los del norte (Baja California, Baja California Sur, Sonora, Nuevo León, Sinaloa, etc.) tienen unas TIC relativamente bien desarrolladas con una alta cobertura de dispositivos electrónicos e Internet, y los estudiantes tienen acceso a una amplia gama de servicios de enseñanza de alta calidad, incluyendo plataformas en línea, programas de televisión, manuales de estudios, etc.; en los estados del sur (Yucatán, Viracruz, Tabasco, Oaxaca, Guerrero, Chiapas, etc.) una parte importante de la población estudiantil tiene acceso a la educación solo a través de manuales de estudio.

Observaciones y perspectivas sobre las modalidades de educación básica en América Latina en la era pandémica y pospandémica-Estudio de caso: Aprende en Casa de México. Xing y Xi. 
RED. Revista de Educación a Distancia. Núm. 67, Vol. 21. Artíc. 9, 30-Sep-2021

DOI: http://dx.doi.org/10.6018/red.480841

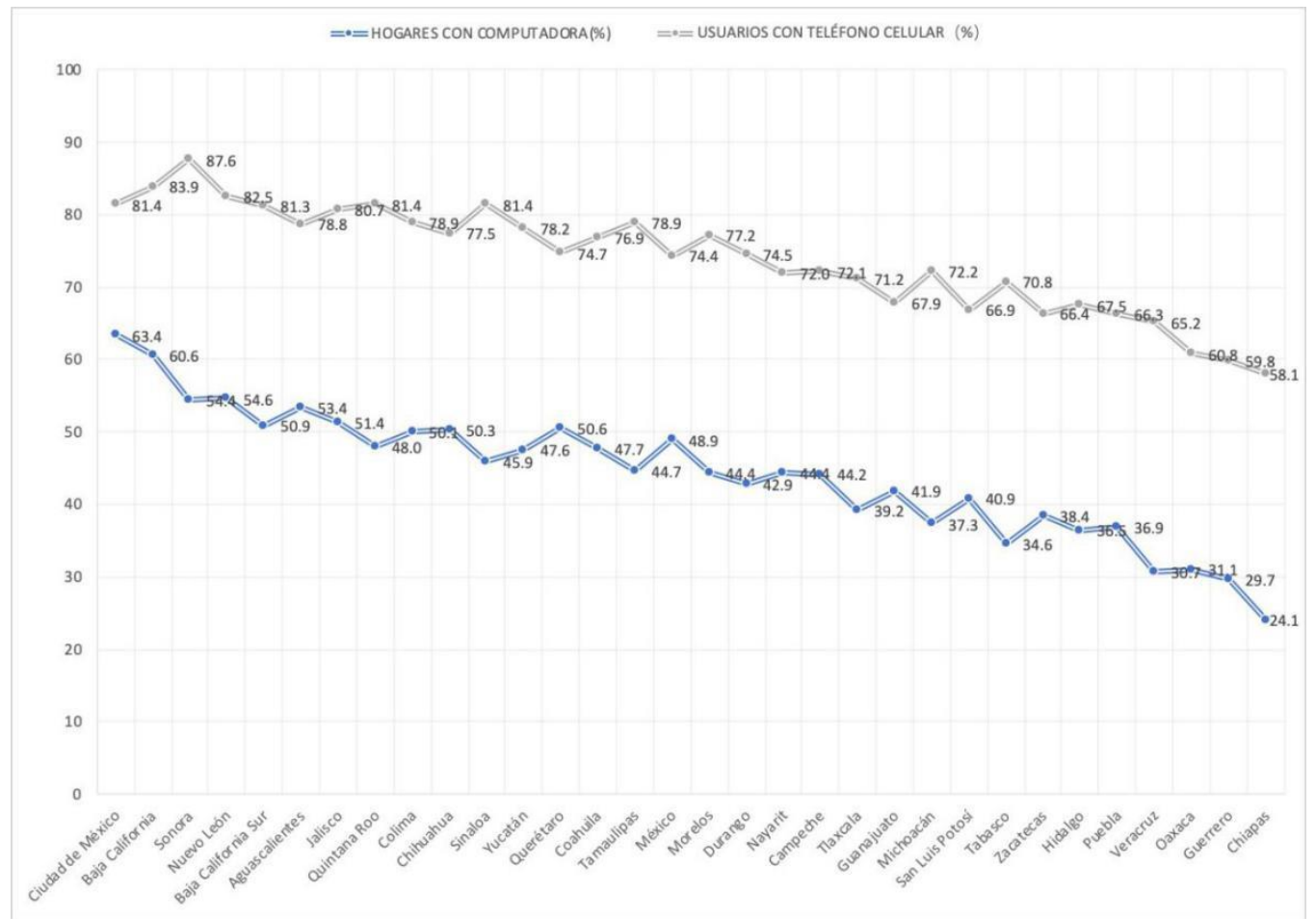

Fig.2 Tasas de disponibilidad de equipos informáticos en los hogares por estado en México, 2018 (SCT, 2020)

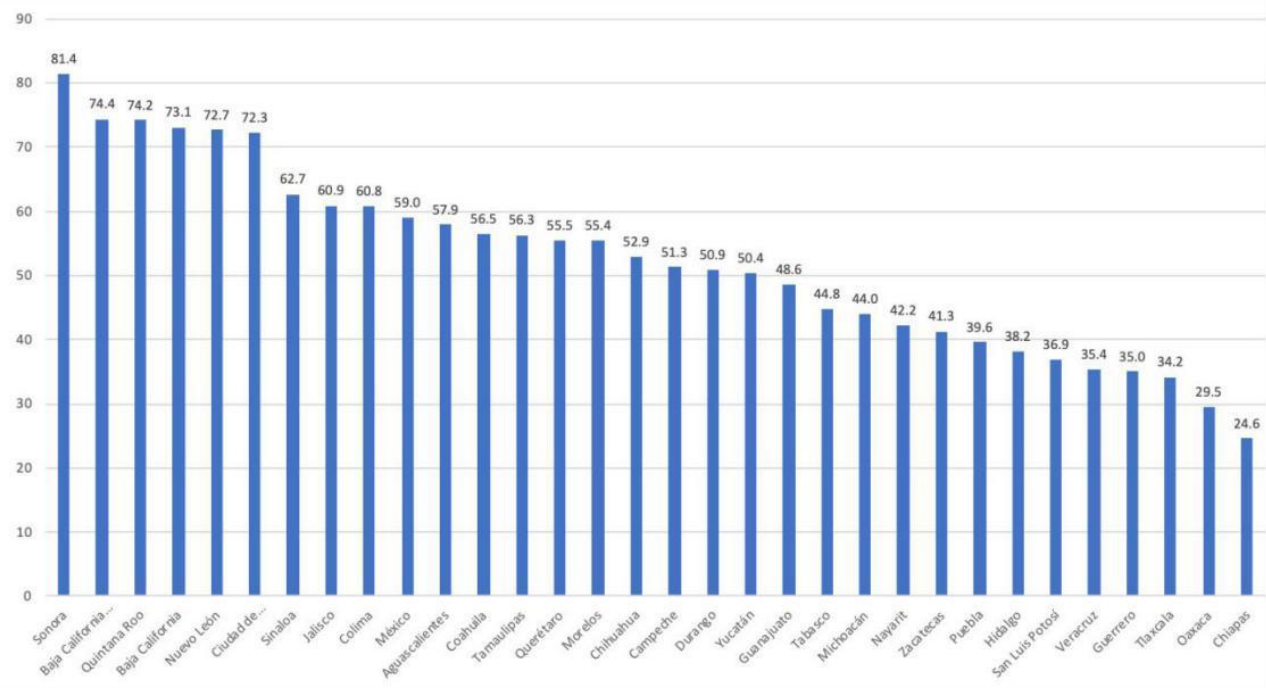

Fig.3 Cobertura de Internet en los hogares por estado en México en 2018 (SCT, 2020)

Desde una perspectiva empírica, la experiencia educativa durante la pandemia confirma esta heterogeneidad regional. Según una encuesta a nivel nacional en abril del año pasado, se observa una escasez en el uso de plataformas digitales en el sur-sureste del país Observaciones y perspectivas sobre las modalidades de educación básica en América Latina en la era pandémica y pospandémica-Estudio de caso: Aprende en Casa de México. Xing y Xi. 
mexicano, lo cual contrasta con la experiencia de la zona norte. La misma diferenciación regional también se manifiesta en la inclinación a recibir una capacitación tecnológica de los docentes, así como en el seguimiento educativo y la comunicación entre el alumnado y el profesorado, siguiendo una tendencia descendente desde el norte hasta el sur (Baptista Lucio, et al, 2020). Por ello, esta brecha pedagógica se evidencia en la modalidad virtual, sobre todo, a lo largo del norte-sur.

En resumen, debido a las limitaciones infraestructurales, la oferta educativa se distribuye de forma desigual entre las regiones, y la gama de productos disponibles y el grado de garantía del derecho a la educación varían de una región a otra, con importantes diferencias regionales. Por lo tanto, aún queda camino por recorrer antes de alcanzar el ideal de "amplia uniformidad" de "equidad en la educación".

\subsection{Escepticismo sobre la eficacia y la calidad de la educación a distancia}

Aunque la educación televisiva es una modalidad emergente en el contexto de la pandemia, el hecho es que el contenido de la enseñanza sigue siendo un "calco" de lo que era antes de la pandemia, todo lo cual no es necesariamente bueno para la reforma del sistema educativo mexicano. Según Ernesto Piedras (Sheila Sánchez, 2020), director general de The Competitive Intelligence Unit, el reto para estas televisoras será el desarrollo de contenidos, ya que tienen que estar muy producidos para que sean muy claros para los estudiantes, además de que no hay interacción. A partir de una comparación transversal internacional, la calidad general de la educación básica en México antes de la era pandémica estaba rezagada, lo que no favorecía el fortalecimiento de la capacidad básica de innovación del país a largo plazo. En el nuevo paradigma educativo bajo la pandemia los problemas inherentes no se han resuelto, mientras que siguen surgiendo otros nuevos: la atención de los alumnos y la eficacia del aprendizaje son muy limitadas en las aulas tradicionales, y es concebible cómo sería la situación en la educación televisada, donde la supervisión y la presión externas son menores. Según la encuesta que realizó la Mejoredu (2020), entre los problemas sobre la educación a distancia expuestos por estudiantes destacan: i) poco acompañamiento o falta de explicaciones de sus docentes; ii) falta de claridad en las actividades; iii) escasa retroalimentación sobre los trabajos realizados; iv) desconocimiento de sus aciertos o errores en las actividades; v) insuficiente comprensión de lo que hacían; vi) menores aprendizaje y comprensión; vii) percepción de no tener los conocimientos necesarios para pasar al siguiente grado.

La educación televisiva exige alta adaptabilidad para la participación de los actores pedagógicos; sin embargo, precisamente su falta de capacidad de respuesta se revela en esta modalidad. Desde el siglo XXI, la profesionalización de la educación ha ido en aumento, y para muchas familias, debido al nivel de conocimientos y a las limitaciones de tiempo y

Observaciones y perspectivas sobre las modalidades de educación básica en América Latina en la era pandémica y pospandémica-Estudio de caso: Aprende en Casa de México. Xing y Xi. 
espacio, la responsabilidad de la educación recae enteramente en la escuela, y los padres también carecen de recursos tecnológicos para mantener el contacto con la escuela (Frank Junior, 2020). En consecuencia, los padres se sienten a menudo abrumados por la forma de sustituir el papel de los profesores y guiar a los alumnos durante este periodo concreto, al carecer de la base de conocimientos adecuada para la tutoría y ser incapaces de aprovechar plenamente los recursos de apoyo proporcionados por la escuela; los profesores, por su parte, se sienten más incómodos por la falta de conocimientos tecnológicos; a su vez, los estudiantes, destinatarios de las actividades docentes, también se enfrentan a problemas de adaptación, aunque se sienten relativamente más cómodos con los dispositivos digitales, que antes de la pandemia se utilizaban principalmente para el entretenimiento y se han transformado repentinamente en herramientas de aprendizaje. Todos los factores arriba mencionados constituyen grandes y nuevos retos y fuentes de preocupación por la calidad educativa bajo la nueva modalidad de Aprende en Casa.

\subsection{Falta de educación social y humanística en la enseñanza televisiva}

Al igual que otros modelos de educación a distancia, la teleducación tiene la desventaja de dejar de lado la importante dimensión interpersonal. Las interacciones sociales entre profesores y alumnos y entre alumnos son importantes para mejorar las percepciones cognitivas de los individuos, aumentar su nivel de adaptación social y conseguir un desarrollo saludable de la personalidad, que es una parte imprescindible de la educación moral (Xiao, 1996). Sin embargo, en esta nueva modalidad la interacción humana es casi nula, lo cual puede provocar una falta de habilidades interpersonales y de cognición social, además del distanciamiento entre profesores y alumnos. La consecuencia será, sin lugar a dudas, la dificultad del proceso de socialización de los alumnos.

Después de la pandemia de SARS, los estudios científicos han demostrado que el aislamiento en el hogar impide en gran medida la comunicación normal entre las personas aisladas y el mundo exterior, lo que provoca miedo, depresión, ansiedad, pesimismo, incertidumbre e incluso desesperación sobre el futuro (Reynolds D1, 2008). Junto con el impacto de diversos factores, tales como la pérdida de seres queridos y las dificultades económicas durante la pandemia, la salud mental se convierte en una preocupación extraordinariamente seria a lo largo y después de la cuarentena. Los datos de la Mejoredu (2020) muestran que casi la mitad de docentes y de estudiantes (48,7 y 49,9\%, respectivamente) a menudo sentían tensión por las actividades académicas que debían atender; la mitad de madres y padres de familia, y estudiantes (50,1 y 49,7\%, respectivamente) con frecuencia sintieron que las actividades que debían realizar eran tantas que no podían terminarlas; y 39,8\% de estudiantes y 41,3\% de madres y padres de familia se sintieron tristes o desanimadas(os) por la situación que vivían. Parte del

Observaciones y perspectivas sobre las modalidades de educación básica en América Latina en la era pandémica y pospandémica-Estudio de caso: Aprende en Casa de México. Xing y Xi. 
estudiantado manifestó estrés y ansiedad derivados de una carga excesiva de tareas; revelaron frustración y enojo por no entender las actividades y por la sensación de no estar aprendiendo bien; también expresaron tristerza por la pérdida de algún ser querido, deseo de reestablecer el vínculo social con otros estudiantes y docentes, y necesidad de recibir atención psicológica; para cierto número de estudiantes los niveles de estrés, ansiedad y depresión resultaban inmanejables.

La investigación regional de Nuevo León (México) también ha dado certeza al hecho de que un porcentaje importante de profesores han tenido sentimientos negativos, entre los cuales se encuentran el estrés por diseñar actividades remotas, la angustia y la consternación. Más de lo mismo pasa en el caso de los alumnos siendo el aburrimiento, la angustia y la tristeza las emociones negativas más preponderantes durante el encierro pandémico.

El secretario general de la ONU, Guterres, también dijo en su informe que la pandemia del covid-19 ha afectado gravemente al bienestar mental de la comunidad humana y que los países deben prestar gran atención para responder de manera oportuna a la crisis de salud mental en estas circunstancias pandémicas (Naciones Unidas, 2020). De hecho, la educación tiene un importante papel social y la responsabilidad de cuidar la salud psicológica de los alumnos. Sin embargo, existe un grado considerable de aislamiento y depresión en el proceso de aprendizaje bajo el modelo de teleducación. Según la encuesta de UNICEF de 2020 sobre las afectaciones por la pandemia de covid-19 en el bienestar de las niñas, niños y adolescentes en México, el 35,8\% de las familias con hijos adolescentes de 0 a 17 años que fueron entrevistadas en mayo de 2020 declararon tener síntomas graves de ansiedad, valor que se redujo al 33,8\% en julio (UNICEF, 2021). Es evidente que ha existido una falta de atención emocional hacia los alumnos y un descuido en la orientación educativa sobre salud psicológica a lo largo de la cuarentena.

\section{Perspectivas sobre el desarrollo de la educación básica en la región latinoamericana en el contexto de la pandemia y la era pospandémica}

La pandemia inició el camino de la transformación digital en el que los estudiantes han pasado de aprender presencialmente a aprender de forma remota. Esto requirió, y va a requerir, ajustes significativos no solo de los estudiantes y maestros, sino también de las familias, los directivos y, en general, de toda la sociedad (Sosa Neira, 2020) El modelo de educación básica en México bajo la pandemia es solo un microcosmos de la situación de

Observaciones y perspectivas sobre las modalidades de educación básica en América Latina en la era pandémica y pospandémica-Estudio de caso: Aprende en Casa de México. Xing y Xi. 
América Latina, y otros países también han revelado problemas similares en diversos grados. Por lo tanto, es necesario romper el viejo patrón educativo en las nuevas circunstancias del covid-19, responder a los problemas y desafíos a los que se enfrenta la educación básica de manera específica en el contexto de la realidad social regional, y abrir perspectivas más amplias para el desarrollo de la educación.

La educación básica en América Latina se enfrenta a dos problemas comunes: por un lado, el desigual desarrollo socioeconómico de la región y la vulnerabilidad de la garantía de la educación básica han provocado un fuerte aumento de la tasa de abandono escolar ante la crisis sanitaria pública; por otro, aunque Chile, Uruguay y Costa Rica se encuentran entre los primeros países de la región en cuanto a la calidad de la educación básica, la alfabetización básica de los alumnos está por debajo de la media mundial, la proporción de alumnos bien formados es baja, y a grandes rasgos, la calidad de la enseñanza es preocupante.

En respuesta a estos dos grandes retos, será indispensable promover la equidad en el acceso y un alto nivel de desarrollo en la educación básica, con el objetivo de lograr un desarrollo sostenible, una interfaz orgánica entre la educación básica y la superior o la formación profesional, así como modernizar el sistema educativo. Para ello, planteamos las siguientes sugerencias con base en las experiencias exitosas de algunos países durante la pandemia:

\section{Aumentar la inversión pública en la educación para garantizar la igualdad de acceso}

Una forma importante de ampliar la cobertura de la educación y promover la igualdad de acceso es aumentar la proporción del gasto público en educación. Según un informe publicado por la CEPAL en 2019, el porcentaje promedio entre el gasto en educación y el PIB en los países latinoamericanos ha seguido una tendencia al alza constante desde principios del siglo y se ha estabilizado en el $4 \%$ entre 2015 y 2018, y varios países de la región han alcanzado el objetivo orientativo de "entre el 4\% y el 6\% del PIB o entre el 15\% y el 20\% del gasto público total en educación", tal y como establece el Marco de Acción de las Naciones Unidas para la Educación 2030 (CEPAL, 2019). América Latina va por el buen camino al lograr algunos avances en el aumento de la cobertura educativa mediante la expansión del gasto público en educación. Sin embargo, en el contexto de la recesión económica global provocada por el coronavirus, lo de cómo garantizar o incluso aumentar el gasto en educación, incrementar la capacidad absoluta del sistema educativo básico y mantener la prioridad en las áreas de inversión pública se ha convertido en una cuestión espinosa.

Un aumento de la inversión financiera no favorece por sí solo la sostenibilidad de la política social; por lo tanto, las transferencias monetarias condicionadas son necesarias para Observaciones y perspectivas sobre las modalidades de educación básica en América Latina en la era pandémica y pospandémica-Estudio de caso: Aprende en Casa de México. Xing y Xi. 
incentivar a los grupos de bajos ingresos a utilizar los servicios públicos, como la educación o la sanidad, mediante subvenciones monetarias condicionadas (Huang, 2020). En el ámbito de la educación, estos programas de asistencia social específicos ayudan a resolver la difícil situación de la baja matriculación y las altas tasas de abandono escolar causadas por las limitaciones económicas, mejorando así el nivel general de la educación. La experiencia de México es pionera en el continente latinoamericano. El programa ProgresaOportunidades-Prospera, puesto en marcha en 1997, condiciona las ayudas económicas del gobierno a las familias pobres a la asistencia a la escuela y a las revisiones médicas periódicas de los niños. El actual presidente López Obrador lo reformó transfiriendo parte de sus fondos al nuevo programa de Becas para el Bienestar Benito Juárez, que cubre la educación básica y el bachillerato, y relajando los requisitos de la solicitud y las condiciones previas para las transferencias de efectivo (Russell, 2019). Este enfoque ha causado cierta controversia en la comunidad escolar. Además de la preocupación de que no sea eficaz para garantizar la permanencia de los niños en la escuela y la mejora de los recursos humanos, algunos estudiosos sostienen que la reducción de los requisitos de las transferencias monetarias no favorece el desarrollo de las competencias cívicas (Schober, 2019).

En conclusión, es tarea de cada país latinoamericano mantener el monitoreo de la efectividad de los programas de transferencias monetarias, ajustando sus condiciones previas a la realidad social y mejorando la sensibilidad regional de la política para hacerlos compatibles con su contexto nacional.

\section{- Reforzar las infraestructuras educativas y promover la homogeneidad de la calidad de la enseñanza}

Las instalaciones educativas geográficamente homogéneas son la base material para lograr la equidad en la calidad de la educación. En el caso de México, por ejemplo, es imprescindible que el Gobierno mejore las infraestructuras y los servicios de agua y electricidad en las escuelas de las zonas desfavorecidas. Al mismo tiempo, también es igualmente importante sincronizar las instalaciones relacionadas con tecnologías de la información y comunicación (TLC). Cabe aclarar que la transformación digital de las instituciones educativas no es convertir la educación presencial en educación a distancia o virtual, sino usar las TLC para mejorar procesos educativos. La actual situación se ha convertido en una fase experimental y en una oportunidad para determinar aquellas experiencias y recursos tecnológicos que sirven para mejorar el aprendizaje de los estudiantes y que se pueden seguir implementando cuando la comunidad educativa regrese a las clases presenciales (Sosa Neira, 2021). Sin embargo, según el informe de abril de 2020 del Banco Interamericano de Desarrollo (Rieble-Aubourg \& Viteri, 2020), un estudio

Observaciones y perspectivas sobre las modalidades de educación básica en América Latina en la era pandémica y pospandémica-Estudio de caso: Aprende en Casa de México. Xing y Xi. 
sobre las condiciones de informatización escolar en 26 países de América Latina muestra que solo Uruguay ha alcanzado el objetivo del proyecto Sistemas de Información y Gestión Educativa (SIGED). En 2018, antes del brote del covid-19, la cobertura media escolar de Internet en esta región era de solo el 33\% y la media de las zonas desfavorecidas se reducía al $22 \%$. En la era de la información es urgente que los gobiernos latinoamericanos den prioridad al desarrollo de infraestructuras de red y equipamiento informático para las escuelas en zonas marginadas.

La mejora de las infraestructuras educativas no solo se refiere a la construcción de infraestructuras escolares, sino también a la provisión de instalaciones informáticas en los hogares. En 2007 Uruguay puso en marcha el Plan Ceibal, un programa de conectividad informática diseñado para la educación en línea, con el fin de proporcionar un ordenador portátil a los estudiantes de las escuelas públicas de todo el país y fortalecer la plataforma digital nacional de educación. La Universidad Nacional Autónoma de México ha llevado a cabo un proyecto similar proporcionando tabletas de préstamo temporal durante la pandemia. Estos dos programas han sido muy eficaces y han tenido mucho éxito durante esta deriva dramática, y son relevantes para la educación básica.

\section{- Reformar el paradigma educativo para adaptarlo a la era pospandémica}

En el contexto de una pandemia en la que no existe un contacto social seguro, la educación a distancia puede ser una alternativa razonable, pero nunca podrá ser una solución permanente que sustituya por completo la enseñanza tradicional presencial en la era pospandémica. El modelo educativo en la época después de la pandemia debería ser uno en el que ambos se complementen y se beneficien mutuamente. Por un lado, la enseñanza en línea permite romper las restricciones de tiempo y uniformidad de contenidos del sistema tradicional de enseñanza en el aula; por otro, la enseñanza presencial tiene un carácter insustituible: las interacciones cara a cara entre el profesorado y el alumnado y las actividades presenciales como debates en grupo son esenciales para el desarrollo general de los alumnos. Por lo tanto, es necesario innovar la forma de enseñanza de la educación básica en la era pospandémica, seguir la tendencia de los tiempos, fomentar la integración de la enseñanza en línea y presencial y promover la reforma de la educación en la era de la información.

La reforma del sistema educativo no solo abarca la transformación de la modalidad de la enseñanza, sino también la modernización del plan curricular y la actualización del marco de contenidos, con el fin de elevar la calidad de la enseñanza en la región, que está por detrás del nivel medio mundial. Para ello, es necesario rediseñar el marco educativo con el fin de hacer hincapié en el vínculo orgánico entre los conocimientos de la materia y su aplicación práctica, y ofrecer al personal docente y directivo formación pertinente y de

Observaciones y perspectivas sobre las modalidades de educación básica en América Latina en la era pandémica y pospandémica-Estudio de caso: Aprende en Casa de México. Xing y Xi. 
calidad para enfrentar situaciones de emergencia. Al mismo tiempo que los profesores y alumnos siguen siendo los actores activos en el aula, se aprovecharán las ventajas únicas de la educación a distancia para mejorar la capacidad de autoaprendizaje, las competencias prácticas, la iniciativa y la creatividad de los alumnos.

\section{- Construir un mecanismo razonable de evaluación y retroalimentación}

La evaluación y la retroalimentación de los resultados educativos facilitan el seguimiento y la mejoría de los objetivos de la enseñanza. Sin embargo, con la integración de la educación a distancia en la enseñanza ordinaria, el sistema de evaluación del proceso enseñanza-aprendizaje, que antes se aplicaba a la enseñanza presencial tradicional, debe adaptarse a los nuevos cambios de la modalidad educativa, y el nuevo mecanismo debe ser sensible a la heterogeneidad de las condiciones de aprendizaje en la era pandémica y pospandémica. En este sentido, debe desarrollarse un sistema de evaluación que registre de forma eficaz y precisa la eficacia y el proceso del aprendizaje en diferentes condiciones para mejorar la sensibilidad a las diferencias en los objetivos educativos y en el sistema de evaluación, así como para diversificar los criterios de evaluación para la formación continua. Aparte de los criterios que regían en el sistema tradicional como entregas de tareas, prácticas de lectura y resolución de problemas matemáticas, habilidades nuevas como la habilidad tecnológica y colaborativa tanto entre los compañeros como en el entorno familiar también han sido factores a tener en consideración para incluir en un mecanismo de evaluación que esté a la altura de la educación futura.

\section{-Dar importancia a la atención humanística y fortalecer la educación psicológica}

De hecho, la pandemia supone una oportunidad para reflexionar sobre la calidad educativa y sobre todo, su desarrollo sostenible en situaciones adversas e inciertas y en los tiempos pospandémicos. En la era prepandémica, la salud mental no estaba integrada orgánicamente con la educación básica. En Chile, por ejemplo, la forma de atención a la salud psicológica más habitual son los servicios de asesoramiento ofrecidos por la escuela, y no existe una forma sistemática y generalizada a nivel nacional diseñada por el Ministerio de Educación. Con el cierre de las escuelas, este tipo de servicios se interrumpieron y fueron sustituidos por la ayuda psicológica en línea. El Instituto Mexicano de la Juventud, en coordinación con la Secretaría de Salud del país, ha coordinado a los profesionales para que proporcionen la orientación psicológica necesaria a través de WhatsApp a los adolescentes del país que les envíen mensajes en busca de ayuda. No obstante, hay que entender que esta atención es paralela al contexto del coronavirus y todavía queda fuera del sistema educativo básico. Por lo tanto, en la era pospandémica, las autoridades sanitarias nacionales y las escuelas deberían colaborar para realizar investigaciones

Observaciones y perspectivas sobre las modalidades de educación básica en América Latina en la era pandémica y pospandémica-Estudio de caso: Aprende en Casa de México. Xing y Xi. 
periódicas sobre la salud psicológica de los alumnos; al mismo tiempo, deberían mejorar el marco de enseñanza desarrollando cursos de educación en salud mental y elevar su prioridad en el sistema educativo.

Aunada a la falta de parte psicológica dentro del sistema educativo, la educación virtual de por sí ya es un factor contribuyente al reto gigantesco de cuidar al bienestar mental de los estudiantes, vinculado significativamente a las interrelaciones socioeducativas. Previo estudio ha confirma que las clases a distancia basadas en las plataformas de comunicación simultánea que permiten la observación de expresión tanto verbal como no verbal entre los participantes son responsables de emociones negativas y malestar social con dificultades técnicas de por medio en el aprendizaje colaborativo (Belli, 2018). Teniendo en cuenta que, en la educación remota, la interacción en tiempo real a través de las plataformas en línea se convertiría en una nueva normalidad, cabe destacar la relevancia del abordaje de la interacción multimodal y la resolución del revés psicológico generado por los conflictos entre compañeros, beneficiando el bienestar del alumnado en favor de las relaciones interpersonales.

Además de la interrelación entre socios, los padres y los docentes constituyen una pieza fundamental en el aspecto socioeducativo. Según el resultado de una encuesta a 17 municipios de Morelos (México) para investigar la percepción del bienestar de los hijos/as durante la educación a distancia en el contexto del Programa Aprende en Casa, un elemento determinante son las relaciones sociales que se establecen, las cuales están dirigidas a las interrelaciones socioeducativas con la familia y los docentes. Por lo tanto, en términos de la atención humanística, es sumamente importante implementar estrategias y prácticas que favorezcan relaciones afectuosas y participativas alineadas con las políticas educativas (Reviero, 2021).

Díez García et al. (2020) afirmó que las cuestiones relacionadas con la percepción del riesgo en esta crisis sanitaria cobran valor a la luz de nuestros usos y prácticas de aplicaciones de mensajería instantánea. En los tiempos de la pos-pandemia, en que la transformación digital se irá haciendo más importante en nuestras prácticas pedagógicas, el contagio emocional a través de diferentes dimensiones comunicativas entre los actores educativos será un nuevo reto.

\section{- Diseñar capacitación tecnológica de calidad para docente y padres en el marco institucional}

La participación familiar y los docentes han adquirido nuevas dinámicas durante la educación remota. En consonancia con el punto anterior, el bienestar estudiantil se asocia directamente con el dominio tecnológico de los padres en el sentido en que este ayuda en gran medida la interacción de sus hijos/as con los compañeros y los docentes (Reviero, Observaciones y perspectivas sobre las modalidades de educación básica en América Latina en la era pandémica y pospandémica-Estudio de caso: Aprende en Casa de México. Xing y Xi. 
2021). En otras palabras, los padres han pasado de mediadores entre la escuela y sus hijos/as a un actor participativo de suma importancia en el proceso pedagógico.

Al margen de la familia, a los docentes también se les percibe como un rol primordial en las instrucciones y el diseño de actividades escolares bajo el esquema remoto. Sin embargo, el rezago de la capacidad tecnológica de los docentes se ha desnudado a la hora de trasladar las actividades pedagógicas y la evaluación al entorno virtual, por lo que hace falta un enfoque de capacitación tecnológica institucionalizado orientado tanto a la comunidad docente como a los padres, reclamo que figura con mucha frecuencia desde ambos sectores, cuya finalidad es conseguir la mejoría constante del manejo de las TLC con fines pedagógicos.

\section{Conclusión}

La educación es un derecho humano fundamental, un medio importante para el crecimiento y desarrollo personal y un precursor del avance social. Tiene un valor estratégico, especialmente para la región latinoamericana, que necesita urgentemente romper el estancamiento del desarrollo.

Bajo el impacto de la nueva pandemia, la mayoría de los países de la región han tenido que cerrar sus escuelas y trasladar sus actividades docentes presenciales a la red. En esta ola de transformación pedagógica, México, teniendo en cuenta la realidad social del país y las tendencias educativas modernas, lanzó el proyecto Aprende en Casa, utilizando la "educación por televisión" como principal enfoque durante la pandemia. El proyecto refleja la orientación educativa homogénea de la Secretaría de Educación Pública de México y su sensibilidad a las diferencias individuales, promoviendo la diversificación de las herramientas de enseñanza y las formas de evaluación, buscando la paridad de acceso y la igualdad de calidad en la educación a distancia en el contexto de la crisis de salud pública, enfatizando así la inclusión de grupos indígenas de estudiantes y la equidad educativa.

Sin embargo, el modelo mexicano también revela ciertas deficiencias, según el marco conceptual descriptivo, una de las cuales consiste en una clara heterogeneidad geográfica en la garantía de la equidad en la educación, tanto en lo que respecta a la garantía del derecho a la educación como a la calidad específica de la educación orientada a las tecnologías de la información y la comunicación (TIC), con diferencias espaciales principalmente entre el norte y el sur. Al analizar el marco conceptual tipológico de la educación básica como bien público, hemos visto que el programa Aprende en Casa presenta un estado diferenciado de oferta de productos y protección de derechos, que aún está lejos del estado idealizado de "amplia uniformidad". Además, este modelo revela la falta de cambios innovadores en el contenido de la enseñanza y el aprendizaje, el

Observaciones y perspectivas sobre las modalidades de educación básica en América Latina en la era pandémica y pospandémica-Estudio de caso: Aprende en Casa de México. Xing y Xi. 
escepticismo sobre la calidad de la educación y la falta de atención a la salud mental de los estudiantes.

Las deficiencias expuestas en el modelo del Estado mexicano son en cierta medida un reflejo del problema general de la educación básica en América Latina. En respuesta a este dilema educativo, los países latinoamericanos deben innovar su pensamiento en la era pandémica y pospandémica, adherirse a los requisitos de la educación moderna como guía y promover la transformación y actualización del sistema marco de la educación básica. En primer lugar, se debe aumentar el gasto público en educación para ampliar el área cubierta por la educación básica y utilizar los programas de asistencia social con transferencias monetarias condicionadas para proporcionar una red de protección precisa para que los niños en los márgenes de la sociedad asistan a la escuela; además, se debe fortalecer las infraestructuras de información social para reducir la brecha digital en la región y perseguir la homogeneización de la calidad de la educación básica; al mismo tiempo, se debe construir urgentemente un sistema de educación dual con la integración orgánica de la educación en línea y presencial, profundizar en la reforma de los programas de enseñanza para adaptarlos a los requisitos del desarrollo de los recursos humanos modernos y establecer un sistema de evaluación educativa razonable basado en ello, así como integrar la educación en salud mental en el sistema educativo. En conclusión, los países latinoamericanos deben optimizar el sistema tradicional de educación básica desde múltiples dimensiones educativas, superar sus limitaciones institucionales y, en definitiva, poner la educación pública de alta calidad a disposición de todos los estudiantes en edad escolar, maximizando los beneficios de la educación y proporcionando una oferta constante de recursos humanos de alta calidad para el desarrollo moderno del país.

Presentación del artículo: 23 de mayo de 2021

Fecha de aprobación: 6 de septiembre de 2021

Fecha de publicación: 30 de septiembre de 2021

Xing y Xi. (2021). Observaciones y perspectivas sobre las modalidades de educación básica en América Latina en la era pandémica y pospandémica-Estudio de caso: Aprende en Casa de México. RED. Revista de educación a distancia, 21(67). http://dx.doi.org/10.6018/red.480841

\section{Financiación}

Este trabajo no ha recibido ninguna subvención específica de los organismos de financiación en los sectores públicos, comerciales o sin fines de lucro.

Observaciones y perspectivas sobre las modalidades de educación básica en América Latina en la era pandémica y pospandémica-Estudio de caso: Aprende en Casa de México. Xing y Xi. 
RED. Revista de Educación a Distancia. Núm. 67, Vol. 21. Artíc. 9, 30-Sep-2021

DOI: http://dx.doi.org/10.6018/red.480841

\section{Referencias}

Amador Bautista, R. (2020). Aprende en casa con \#SanaDistancia en tiempos de \#COVID-19. En Educación y pandemia : una visión académica (págs. 138-144). Universidad Nacional Autónoma de México, Instituto de Investigaciones sobre la Universidad y la Educación. Recuperado de http://132.248.192.241:8080/xmlui/bitstream/handle/IISUE_UNAM/549/AmadorR_20 20_Aprende_en_casa.pdf?sequence $=1 \&$ isAllowed $=\mathrm{y}$

Baptista Lucio, P., Almazán Zimerman, A., \& Loeza Altamirano, C. A. (2020). Encuesta Nacional a Docentes ante el COVID-19. Retos para la educación a distancia. Revista Latinoamericana De Estudios Educativos, 50(ESPECIAL), 41-88. https://doi.org/10.48102/rlee.2020.50.ESPECIAL.96

Belli, S. (2018). Managing Negative Emotions in Online Collaborative Learning. A multimodal approach to solving technical difficulties. Digithum. A Relational Perspective on Culture and Society, 22.

Campa Álvarez, R. de los Ángeles. (2021). Estrategias y retos para el seguimiento educativo en primarias ante la contingencia covid-19 en Sonora, México. RIDE Revista Iberoamericana Para La Investigación Y El Desarrollo Educativo, 11(22). https://doi.org/10.23913/ride.v11i22.951

CEPAL. (2019). Panorama Social de América Latina 2019. Recuperado de https://www.cepal.org/es/publicaciones/44969-panorama-social-america-latina-2019

Colli, E. C. (2020). Aprende en Casa II. Recuperado de https://www.researchgate.net/publication/344313030_Aprende_en_Casa_II

Comisión Nacional para la Mejora Continua de la Educación (2020). Experiencias de las comunidades educativas durante la contingencia sanitaria por covid-19. Educación básica. Informe ejecutivo. Ciudad de México: autor.

Correa Terán, J. (2021). Un primer análisis de "Aprende en Casa II" - Educación Futura. Recuperado 8 August 2021, de https://www.educacionfutura.org/un-primer-analisis-deaprende-en-casa-ii/

Díez García, R., Belli, S., \& V. Márquez, I. (2020). La COVID-19, pantallas y reflexividad social. Cómo el brote de un patógeno está afectando nuestra cotidianidad. Revista Española De Sociología, 29(3).

Durand, B. G. (24 de diciembre de 2020). Educación y tecnología, los otros rezagos que la pandemia evidenció. Recuperado de El universal:

Observaciones y perspectivas sobre las modalidades de educación básica en América Latina en la era pandémica y pospandémica-Estudio de caso: Aprende en Casa de México. Xing y Xi.

Página 21 de $\mathbf{2 4}$ 
https://www.eluniversal.com.mx/ciencia-y-salud/educacion-y-tecnologia-los-otrosrezagos-que-la-pandemia-evidencio

Frank Junior, H. T. (2020). La Educación en Tiempos de Pandemia: Los Desafíos de la Escuela del Siglo XXI. Venezuela: Revista arbitrada del Centro de Investigaciones y Estudios Gerenciales, págs. 6-8. Recuperado de http://www.grupocieg.org/archivos_revista/Ed.44(176187)\%20Hurtado\%20Tavalera_articulo_id650.pdf

García-Leal, Mariel, Medrano-Rodríguez, Hernán, Vázquez-Acevedo, José Antonio, Romero-Rojas, José Carlos, \& Berrún-Castañón, Luz Natalia. (2021). Experiencias docentes del uso de la tecnología educativa en el marco de la pandemia por COVID19. Revista Información Científica, 100(2), e3436. Epub 01 de marzo de 2021. $\begin{array}{llllll}\text { Recuperado en } 08 \text { de } & \text { agosto de }\end{array}$ http://scielo.sld.cu/scielo.php?script=sci_arttext\&pid=S102899332021000200015\&lng=es\&tlng=es.

Harbers, I., \& Steele, A. (2020). Subnational Variation Across States: A Typology and Research Agenda. Latin American Politics and Society, 62(3), 1-18. doi:10.1017/lap.2020.4

Huang, L. P. (2020). La igualdad educativa entre los géneros en América Latina en el marco de los objetivos de la EPT y el ODS4 de la ONU. Revista de Estudios Latinoamericanos, 042(001), pp. 138-153.

INEGI. (2015). Presentación de la Encuesta Intercensal- Principales resultados. Recuperado de https://www.inegi.org.mx/contenido/productos/prod_serv/contenidos/espanol/bvinegi/p roductos/nueva_estruc/inter_censal/estados2015/702825079697.pdf

INEGI. (2020). Encuesta para la Medición del Impacto COVID-19 en la Educación (ECOVID-ED) $2020 . \quad$ Recuperado de https://www.inegi.org.mx/investigacion/ecovided/2020/

Naciones Unidas. (13 de 5 de 2020). Los servicios de salud mental son parte esencial en todas las respuestas de los gobiernos al COVID-19. Recuperado de Naciones Unidas: https://www.un.org/es/coronavirus/articles/servicios-de-salud-mental-respuestagobiernos-covid-19

Pérez-Archundia, E. (2020). Desigualdad y rezago. El sistema educativo mexicano al desnudo frente a la pandemia del COVID-19. Entramados : educación y sociedad, 7(7), 36-41. Recuperado de https://fh.mdp.edu.ar/revistas/index.php/entramados/article/view/4212

Observaciones y perspectivas sobre las modalidades de educación básica en América Latina en la era pandémica y pospandémica-Estudio de caso: Aprende en Casa de México. Xing y Xi. 
Reynolds D1, G. J. (2008). Understanding, compliance and psychological impact of the SARS quarantine experience. Epidemiology \& Infection, 136(7), pp. 997-1007. doi:https://doi.org/10.1017/S0950268807009156

Rieble-Aubourg, S., \& Viteri, A. (2020). Covid-19: ¿Estamos preparados para el aprendizaje en línea? Banco Interamericano de Desarrollo. Recuperado de https://publications.iadb.org/publications/spanish/document/Nota-CIMA--20-COVID19-Estamos-preparados-para-el-aprendizaje-en-linea.pdf

Ríos, V. (10 de agosto de 2020). La violencia que viene. Recuperado de Expansión Política: https://politica.expansion.mx/voces/2020/08/10/violencia-que-viene

Rivero Espinosa, E., \& Bahena Rivera, A. (2021). Interrelaciones socioeducativas, educación en línea y bienestar durante el confinamiento por Covid-19. Revista Prisma Social, (33), pp. 119-136.

Russell, B. (2019, February 25). What AMLO's Anti-Poverty Overhaul Says About His Government. Recuperado de Americas Quarterly: https://www.americasquarterly.org/article/what-amlos-anti-poverty-overhaul-saysabout-his-government/

Schober, G. S. (2019). Conditional Cash Transfers, Resources, and Political Participation in Latin America. Latin American Research Review, 54(3), p. 591.

SCT. (2020). Las TIC en México. Indicadores 2018. Secretaría de Comunicaciones y Transportes Gobierno de México. Recuperado de https://amiti.org.mx/wpcontent/uploads/2020/01/Indicadores_ENDUTIH_2018.pdf

SEP. (2013). Programa Sectorial de Educación 2013-2018. Gobierno de México. Recuperado de https:/www.gob.mx/sep/documentos/programa-sectorial-de-educacion2013-2018-17277

SEP. (2020). Aprende en casa: orientaciones para fortalecer las estrategias de educación a distancia durante la emergencia por covid-19. Recuperado de https://www.usebeq.edu.mx/PaginaWEB/Content/AprendeEnCasa/Docentes/05Aprende-en-casa-Orientaciones-20-ABRIL-vf\%20(1).pdf

SEP. (2020). Principales Cifras Del Sistema Educativo Nacional 2018-2019. Gobierno de México. Recuperado de https://www.planeacion.sep.gob.mx/Doc/estadistica_e_indicadores/principales_cifras/p rincipales_cifras_2018_2019_bolsillo.pdf

Sheila Sánchez, L. F. (5 de agosto de 2020). Los retos de la televisión para educar a distancia en tiempos de Covid-19. Recuperado de Forbes México:

Observaciones y perspectivas sobre las modalidades de educación básica en América Latina en la era pandémica y pospandémica-Estudio de caso: Aprende en Casa de México. Xing y Xi. 
RED. Revista de Educación a Distancia. Núm. 67, Vol. 21. Artíc. 9, 30-Sep-2021

DOI: http://dx.doi.org/10.6018/red.480841

https://www.forbes.com.mx/negocios-retos-television-educar-distancia-covid-19/

Sosa Neira, E. A. (2021). Percepciones de los estudiantes sobre la estrategia Aprende en Casa durante la pandemia COVID-19. Academia Y Virtualidad, 14(1), 133-150.

UNESCO. (1976). International Standard Classification of Education (ISCED). Recuperado de https://unesdoc.unesco.org/ark:/48223/pf0000020992

UNICEF. (2020). Encuesta \#ENCOVID19Infancia: Efectos de COVID-19 en el bienestar de las niñas, niños y adolescentes. UNICEF México. Recuperado de https://www.unicef.org/mexico/media/4496/file/Acumulado\%20de\%20mayo\%20a\%20 julio.pdf

Xiao, S. M. (1996). Un primer análisis de la estructura interpersonal durante el proceso educativo. Revista Tangdu, 12(01), pp. 83-87.

Observaciones y perspectivas sobre las modalidades de educación básica en América Latina en la era pandémica y pospandémica-Estudio de caso: Aprende en Casa de México. Xing y Xi. 